\title{
FACTOR: A computer program to fit the exploratory factor analysis model
}

\author{
URBANO LORENZO-SEVA and PERE J. FERRANDO \\ Universitat Rovira $i$ Virgili, Tarragona, Spain
}

\begin{abstract}
Exploratory factor analysis (EFA) is one of the most widely used statistical procedures in psychological research. It is a classic technique, but statistical research into EFA is still quite active, and various new developments and methods have been presented in recent years. The authors of the most popular statistical packages, however, do not seem very interested in incorporating these new advances. We present the program FACTOR, which was designed as a general, user-friendly program for computing EFA. It implements traditional procedures and indices and incorporates the benefits of some more recent developments. Two of the traditional procedures implemented are polychoric correlations and parallel analysis, the latter of which is considered to be one of the best methods for determining the number of factors or components to be retained. Good examples of the most recent developments implemented in our program are (1) minimum rank factor analysis, which is the only factor method that allows one to compute the proportion of variance explained by each factor, and (2) the simplimax rotation method, which has proved to be the most powerful rotation method available. Of these methods, only polychoric correlations are available in some commercial programs. A copy of the software, a demo, and a short manual can be obtained free of charge from the first author.
\end{abstract}

Exploratory factor analysis (EFA) has had its ups and downs and its share of criticism. However, it is still one of the most widely used statistical procedures in psychological research. Most criticisms of EFA do not challenge the fundamental usefulness of the technique per se, but rather the manner in which it has been applied in some empirical studies. Part of the blame for this may lie in the way in which the technique is implemented in the most commonly available statistical software (Fabrigar, Wegener, MacCallum, \& Strahan, 1999).

EFA involves taking a series of steps that can be approached using various procedures. The main steps are (1) to estimate or extract the factors, (2) to decide how many factors to retain, (3) to rotate factors to an interpretable orientation, and (4) to obtain individual factor scores. Several traditional procedures for these steps are available in popular statistical packages. However, in general they are very few and, in some cases, far from ideal. This limitation was pointed out by MacCallum as early as 1983 and is still present in modern statistical packages. The makers of these programs have made them easier to use but have not improved the number of indices and procedures implemented.

A review of the specialized literature shows that statistical research into EFA is still quite active and that various

This research was supported by Grant SEC2001-3821-C05-C02 from the Spanish Ministry of Science and Technology with the collaboration of the European Fund for the Development of Regions. Correspondence concerning this article should be addressed to U. Lorenzo-Seva, Universitat Rovira i Virgili, Departament de Psicología, Ctra. De Valls, s/n, 43007 Tarragona, Spain (e-mail: urbano.lorenzo@urv.cat). new developments and methods have been presented in recent years. Given that the creators of the most popular statistical packages do not seem very interested in incorporating these new advances, most developers offer programs or macros that allow these recent and specific procedures to be computed. This solves the problem partially, but because different programs must be used for the different procedures, and because these programs are scattered around the literature, it is unlikely that the new methods will be popularized. It may be that if a particular EFA procedure is not offered in a current popular program, or at least in a general program, a researcher is unlikely to use it.

An example of a program that computes procedures and indices not included in any commercial statistical package is the comprehensive exploratory factor analysis (CEFA) pack developed by Browne, Cudeck, Tateneni, and Mels (2004). This allows one, for example, to compute McKeon's (1968) Infomax rotation or Yates's (1987) Geomin rotation. Browne (2001) compared these rotation criteria to other well-known criteria and showed their high efficiency. However, none of the commercial programs implement them.

In this article, we present the program FACTOR, which was designed as a general, user-friendly program for computing EFA. FACTOR uses traditional procedures and indices and incorporates the benefits of more recent developments not included in any commercial package or CEFA pack.

FACTOR is developed to fit the EFA model. Below, we describe in detail the procedures used.

Factor analysis can be computed from different kinds of dispersion matrices, including covariance matrices, 
Pearson correlation matrices, and tetrachoric/polychoric correlation matrices. The suitability of the dispersion matrix to be factor analyzed is assessed by three tests: the determinant of the matrix, Bartlett's test, and the KaiserMeyer-Olkin index.

FACTOR requires the number of factors (or components) to be retained to be specified. However, the minimum average partial test (Velicer, 1976) and parallel analysis (Horn, 1965) can be computed to help determine whether or not the number of dimensions retained was suitable. Also, the eigenvalues of the dispersion matrices are printed. This means that Cattell's screen test can be applied.

Both principal component analysis (PCA) and factor analysis can be computed. For factor analysis, the program uses two common procedures - unweighted least squares (ULS) and exploratory maximum likelihood (EML) - plus a relatively new and unusual procedure: minimum rank factor analysis (MRFA; see, e.g., Shapiro $\&$ ten Berge, 2002; ten Berge \& Kiers, 1991). The MRFA method decomposes observed variables into common parts and unique parts that satisfy the following classical requirements: The covariance matrices for common and unique parts must be positive semidefinite (no negative eigenvalues), and the latter covariance matrix must be diagonal. Subject to these requirements, the method minimizes the common variance that is ignored when only some factors are maintained. The proportion of common variance explained by each of the retained factors can then be interpreted. In ULS, the Heywood correction described in Mulaik (1972, p. 153) is included: When an update has a sum of squares larger than the observed variance of the variable, the corresponding row is updated by constrained regression using the procedure proposed by ten Berge and Nevels (1977).

When ULS or EML is computed, the program returns the following indices to assess the model's goodness of fit: (1) the goodness-of-fit chi-square statistic; (2) the nonnormed fit index, also known as the Tucker-Lewis reliability coefficient; (3) the comparative fit index; (4) the goodness-of-fit index; (5) the adjusted goodness-of-fit index; (6) the root mean square error of approximation; and (7) the estimated noncentrality parameter. Descriptive statistics of the distribution of residuals are also computed.

After the extraction phase, the solution is rotated to achieve maximum simplicity and interpretability. The program includes a large number of rotation methods. Some of these methods, such as varimax (Kaiser, 1958), direct oblimin (Clarkson \& Jennrich, 1988), and promax (Hendrickson \& White, 1964), are already very popular in the research literature, whereas others are new. One of the most efficient rotation methods ever proposed is probably simplimax (Kiers, 1994). Unfortunately, this method is a little difficult to use: An interval of possible salient loadings must be specified, and a final one must be selected. The program suggests reasonable values (following the advice of Kiers, 1994) that usually lead to good results.

Alternatively, promin (Lorenzo-Seva, 1999) can be computed. This special case of simplimax usually leads to good results and does not require any parameter to be specified. Another recent method is weighted oblimin (Lorenzo-Seva, 2000), which is intended to detect the simplest observed variables before any rotation is performed, and these variables lead to the final position of the rotated axes. Finally, it must be pointed out that the following advice, offered by Browne (2001), is implemented in any rotation: (1) To avoid convergence to local maxima, each rotation is computed from a number of random starts and from a prerotation method (clever start), and the rotated solution that attains the optimal criterion value is taken as the solution for the analysis; and (2) Kaiser's (1958) normalized weights and Cureton and Mulaik's (1975) weights are available. Even if these recommendations are implemented by default, they can also be modified by the user.

After the rotation phase, FACTOR returns a series of indices - namely, (1) the reliabilities of orthogonally rotated components (ten Berge \& Hofstee, 1999); (2) the reliabilities of rotated factor scores by the formula $1 /\left(1+S E^{2}\right)$ given by Mislevy and Bock (1990), where $S E$ is the standard error of factor scores; (3) the proportion of common variance explained by each rotated factor when MRFA is computed; (4) Bentler's (1977) simplicity; and (5) the loading simplicity indices (Lorenzo-Seva, 2003) - to assess the level of factor simplicity attained in the rotated solution.

Finally, factor scores are computed by the linear prediction method originally proposed by Anderson and Rubin (1956; see Gorsuch, 1983), which was generalized to oblique factor solutions by McDonald (1981) and further developed by ten Berge, Krijnen, Wansbeek, and Shapiro (1999).

\section{Input and Output}

The input consists of an ASCII format file containing respondents' scores, the number of participants and variables, and the number of factors expected. Alternatively, an ASCII format file containing the covariance or correlation matrix can be used. When simplimax is computed, further input is required. This is the number of expected salient values in the rotated loading matrix (i.e., loadings whose values are clearly different from zero). Even if a number of salient loadings are expected in the rotated loading matrix, simplimax encourages the user to check an interval of salient loadings rather than just one value. The program suggests the values of these intervals. Since a final number must be selected, the program follows Kiers's (1994) advice - that is, it looks out for the largest jump in simplimax function and suggests this value.

The output consists of the indices explained above and is stored in the ASCII format file Output.txt. Even if the output information is too detailed, the user can choose a simplified output.

\section{An Empirical Example}

The accuracy of FACTOR is illustrated by an empirical example. We used two sets of items selected from the Eysenck Personality Questionnaire Revised (Eysenck, Eysenck, \& Barrett, 1985). The first set consisted of 8 binary 
items taken from the Extraversion scale. The second set consisted of 6 binary items taken from the Neuroticism scale. These 14 items were mixed and presented in a single questionnaire. Respondents were 279 psychology and social sciences undergraduates at a university in Spain. The theory suggested that a two-factor model should be expected.

With this data set, we computed PCA, ULS, and EML using our program FACTOR, but also using SPSS, SAS, BMDP, and CEFA. To obtain comparable outputs from the different programs, we always computed the Pearson correlation matrix, retained two factors (or components), and rotated the data using direct oblimin $(\gamma=0)$. We also rotated the data using normalized promax $(k=4)$ to allow comparison with SAS output. Tucker's (1951) congruence coefficients between factors (or components) obtained by FACTOR and the other programs are shown in Table 1.

The high congruence of the values shown in Table 1 indicates that the solutions obtained by FACTOR were identical to those obtained by the other programs, so the interpretation of the factor solutions was also identical irrespective of the program used.

Finally, we attempted an analysis that cannot be obtained using SPSS, SAS, BMDP, CEFA, or other commercial programs. We analyzed polychoric correlations, extracted factors using MRFA, and computed simplimax oblique rotation. Table 2 shows the eigenvalues of the reduced correlation matrix, the proportion of common variance explained by each eigenvalue, the total amount of common variance, and the proportion of common variance explained by the two factors retained. We can see that none of the eigenvalues is negative (as would be the case if any other extraction method were used), so the total amount of common variance (10.911) and the corresponding proportion of common variance (.632) explained by the two factors extracted are available.

Table 3 shows the rotated loading matrix, the commonality of each item, the common variance explained by each rotated factor (and its corresponding proportion), and the reliabilities of the rotated factor scores. The level of factor simplicity attained after rotation was assessed by the values of Bentler's (1977) simplicity index and the loading simplicity index, which were .998 and .707 , respectively. Both values were highly significant, since they were in the 99th percentile in the distribution of possible values for this data set. The interfactor correlation value was .283.

Table 1

Congruence Coefficients Between Factors (or Components) Obtained by FACTOR and by Other Programs

\begin{tabular}{lcccccccc}
\hline & \multicolumn{2}{c}{ PCA } & & \multicolumn{2}{c}{ ULS } & & \multicolumn{2}{c}{ EML } \\
\cline { 2 - 3 } Program & CI & CII & & FI & FII & & FI & FII \\
\hline SPSS* & 1.000 & 1.000 & & 1.000 & 1.000 & & 1.000 & 1.000 \\
CEFA* $^{n}$ & - & - & & 1.000 & 0.998 & & 1.000 & 0.998 \\
SAS** & 1.000 & 1.000 & & 1.000 & 1.000 & & 1.000 & 1.000 \\
BMDP* $^{*}$ & 1.000 & 1.000 & & - & - & & 1.000 & 0.998 \\
\hline
\end{tabular}

Note-CI and FI, extraversion; CII and FII, neuroticism. * ${ }^{*}$ Direct obli$\min (\gamma=0)$ computed. ${ }^{* *}$ Promax $(k=4)$ computed.
Table 2

Eigenvalues and Proportions of Common Variance Obtained by MRFA

\begin{tabular}{cccc}
\hline Factor & Eigenvalue & $\begin{array}{c}\text { Proportion of } \\
\text { Common Variance }\end{array}$ & $\begin{array}{c}\text { Accumulated Proportion } \\
\text { of Variance }\end{array}$ \\
\hline 1 & 4.499 & .412 & .412 \\
2 & 2.396 & .220 & .632 \\
3 & 1.068 & .098 & \\
4 & 0.716 & .066 & \\
5 & 0.630 & .058 & \\
6 & 0.572 & .052 & \\
7 & 0.407 & .037 & \\
8 & 0.250 & .023 & \\
9 & 0.156 & .014 & \\
10 & 0.123 & .011 & \\
11 & 0.063 & .006 & \\
12 & 0.031 & .003 & \\
13 & 0.000 & .000 & \\
14 & 0.000 & .000 & \\
\hline
\end{tabular}

Note-Total common variance $=10.911$.

\section{Program Limitations}

We have developed FACTOR in Visual $\mathrm{C}++$ to be run in Microsoft Windows operating systems. We have tested the program on several computers with different chips (always Pentium) and versions of Windows $(95,98$, NT, 2000, and XP) and found that it works correctly.

The number of variables and subjects in the data set is not limited. However, when large data sets are analyzed, the speed of the analysis depends on the amount of memory installed in the computer. One limitation of FACTOR could be the time needed to compute some of the methods used, such as MRFA or simplicity indices.

Although they can be considered the exception rather than the rule, convergence problems and improper solutions could appear in practical applications and have been reported in relation to ill-conditioned, nearly singular matrices. Commercially available programs usually have a set of automatic, internal procedures (modifications, corrections, or restrictions) for dealing with such problems (see, e.g., Clarke, 1970; Jöreskog, 1977), and an acceptable final solution is usually found. In our opinion, however, a convergence problem or an improper solution must be considered an indication that there is some problem with the data or that the hypothesized factor model is inappropriate for the population being sampled. Therefore, no such automatic controls have been programmed. Examples of situations in which FACTOR fails to converge are cases in which (1) the correlation matrix is not positive definite, and therefore MRFA cannot be computed; and (2) the determinant of the dispersion matrix is lower than .00001, and therefore no further analysis will be computed.

Finally, FACTOR computes neither any available extraction method nor loading standard errors. It must be said that loading standard errors can be computed using CEFA pack.

\section{Program Availability}

A copy of the software, a demo, and a short manual can be obtained free of charge from the first author at urbano .lorenzo@urv.cat. 
Table 3

Oblique Loading Matrix After Simplimax Rotation

\begin{tabular}{lrr}
\hline & \multicolumn{1}{c}{ Item } & \multicolumn{1}{c}{ FII } \\
\hline E1 & $\mathbf{0 . 6 5 2}$ & 0.278 \\
E2 & $\mathbf{0 . 6 2 0}$ & -0.125 \\
E3 & $\mathbf{0 . 8 8 1}$ & -0.102 \\
E4 & $\mathbf{0 . 7 9 1}$ & -0.090 \\
E5 & $\mathbf{0 . 4 5 6}$ & 0.137 \\
E6 & $\mathbf{0 . 7 4 7}$ & 0.007 \\
E7 & $\mathbf{0 . 5 5 5}$ & 0.014 \\
E8 & $\mathbf{0 . 5 9 7}$ & -0.005 \\
N1 & 0.010 & $\mathbf{0 . 8 8 1}$ \\
N2 & -0.023 & $\mathbf{0 . 5 3 3}$ \\
N3 & 0.042 & $\mathbf{0 . 6 3 5}$ \\
N4 & -0.016 & $\mathbf{0 . 6 9 7}$ \\
N5 & 0.178 & $\mathbf{0 . 6 6 8}$ \\
N6 & -0.116 & $\mathbf{0 . 8 0 5}$ \\
Explained common variance & 3.706 & 3.188 \\
Proportion of explained & & \\
common variance & .340 & .292 \\
Reliability & .898 & .895 \\
\hline
\end{tabular}

Note-Absolute values higher than 0.30 are shown in boldface.

\section{REFERENCES}

Anderson, R. D., \& Rubin, H. (1956). Statistical inference in factor analysis. In Proceedings of the 3rd Berkeley Symposium of Mathematical Statistics and Probability (Vol. 5, pp. 111-150). Berkeley: University of California Press.

Bentler, P. M. (1977). Factor simplicity index and transformations. Psychometrika, 42, 277-295.

Browne, M. W. (2001). An overview of analytic rotation in exploratory factor analysis. Multivariate Behavioral Research, 36, 111-150.

Browne, M. W., Cudeck, R., Tateneni, K., \& Mels, G. (2004). CEFA: Comprehensive Exploratory Factor Analysis, Version 2.00 [Computer software and manual]. Available at http://quantrm2.psy .ohio-state.edu/browne/.

Clarke, M. R. B. (1970). A rapidly convergent method for maximumlikelihood factor analysis. British Journal of Mathematical \& Statistical Psychology, 23, 43-52.

Clarkson, D. B., \& Jennrich, R. I. (1988). Quartic rotation criteria and algorithms. Psychometrika, 53, 251-259.

Cureton, E. E., \& Mulaik, S. A. (1975). The weighted varimax rotation and the promax rotation. Psychometrika, 40, 183-195.

Eysenck, S. B. G., Eysenck, H. J., \& Barrett, P. T. (1985). A revised version of the Psychoticism scale. Personality \& Individual Differences, 6, 21-29.

Fabrigar, L. R., Wegener, D. T., MacCallum, R. C., \& Strahan, E. J. (1999). Evaluating the use of exploratory factor analysis in psychological research. Psychological Methods, 4, 272-299.

Gorsuch, R. L. (1983). Factor analysis. Hillsdale, NJ: Erlbaum.
Hendrickson, A. E., \& White, P. O. (1964). Promax: A quick method for rotation to oblique simple structure. British Journal of Statistical Psychology, 17, 65-70.

HorN, J. L. (1965). A rationale and test for the number of factors in factor analysis. Psychometrika, 30, 179-185.

JöRESKOG, K. G. (1977). Factor analysis by least-squares and maximumlikelihood methods. In K. Enslein, A. Ralston, \& H. S. Wilf (Eds.), Statistical methods for digital computers (pp. 125-153). New York: Wiley.

KAISER, H. F. (1958). The varimax criterion for analytic rotation in factor analysis. Psychometrika, 23, 187-200.

Kiers, H. A. L. (1994). Simplimax: An oblique rotation to an optimal target with simple structure. Psychometrika, 59, 567-579.

Lorenzo-Seva, U. (1999). Promin: A method for oblique factor rotation. Multivariate Behavioral Research, 34, 347-356.

Lorenzo-Seva, U. (2000). The weighted oblimin rotation. Psychometrika, 65, 301-318.

Lorenzo-Seva, U. (2003). A factor simplicity index. Psychometrika, 68, 49-60.

MacCallum, R. (1983). A comparison of factor analysis programs in SPSS, BMDP, and SAS. Psychometrika, 48, 223-231.

McDonald, R. P. (1981). Constrained least squares estimators of oblique common factors. Psychometrika, 46, 337-341.

McKeon, J. J. (1968). Rotation for maximum association between factors and tests. Unpublished manuscript, George Washington University.

Mislevy, R. J., \& Bock, R. D. (1990). BILOG 3: Item analysis and test scoring with binary logistic models. Mooresville, IN: Scientific Software.

MulaIK, S. A. (1972). The foundations of factor analysis. New York: McGraw-Hill.

Shapiro, A., \& ten Berge, J. M. F. (2002). Statistical inference of minimum rank factor analysis. Psychometrika, 67, 79-94.

ten Berge, J. M. F., \& Hofstee, W. K. B. (1999). Coefficients alpha and reliabilities of unrotated and rotated components. Psychometrika, 64, 83-90.

TEN Berge, J. M. F., \& Kiers, H. A. L. (1991). A numerical approach to the exact and the approximate minimum rank of a covariance matrix. Psychometrika, 56, 309-315.

ten Berge, J. M. F., Krijnen, W., Wansbeek, T., \& Shapiro, A. (1999). Some new results on correlation-preserving factor scores prediction methods. Linear Algebra \& Its Applications, 289, 311-318.

ten Berge, J. M. F., \& Nevels, K. (1977). A general solution to Mosier's oblique Procrustes problem. Psychometrika, 42, 593-600.

TUCKER, L. R. (1951). A method for synthesis of factor analysis studies (Personnel Research Section Report No. 984). Washington, DC: U.S Department of the Army.

VELICER, W. F. (1976). Determining the number of components from the matrix of partial correlations. Psychometrika, 41, 321-327.

Yates, A. (1987). Multivariate exploratory data analysis: A perspective on exploratory factor analysis. Albany: State University of New York Press.

(Manuscript received February 2, 2004; revision accepted for publication March 28, 2005.) 